\title{
Thermoluminescence studies in swift heavy ion irradiated aluminum oxide
}

\author{
K.R. Nagabhushana ${ }^{a}$, B.N. Lakshminarasappa ${ }^{a, *}$, Fouran Singh ${ }^{b}$ \\ ${ }^{a}$ Department of Physics, Jnanabharathi Campus, Bangalore University, Bangalore 560 056, India \\ ${ }^{\mathrm{b}}$ Inter University Accelerator Centre, P.O. Box No. 10502, New Delhi 110 067, India
}

\begin{abstract}
Thermoluminescence (TL) of combustion-synthesized aluminum oxide bombarded with $120 \mathrm{MeV}$ swift $\mathrm{Au}^{9+}$ ions in the fluence range of $1 \times 10^{11}-2 \times 10^{13}$ ions $\mathrm{cm}^{-2}$ has been studied at room temperature. Two TL glows-a well-resolved one with peak at $\sim 623 \mathrm{~K}(\mathrm{Tg} 2)$ and another unresolved one at $\sim 513 \mathrm{~K}(\mathrm{Tg} 1)$ - are recorded at a heating rate of $10 \mathrm{~K} \mathrm{~s}^{-1}$. It is found that the TL intensity increases with the fluence up to $1 \times 10^{13}$ ions $\mathrm{cm}^{-2}$ and then decreases with increase in fluence. Also, the prominent glow peak temperature $\left(\mathrm{Tg}_{2}\right)$ is found to be shifted towards the lower temperature region, while the TL intensity increases with the increase in ion fluence. In the case of heat-treated samples, the TL intensity is observed to be enhanced further. However, in the case of samples heat-treated beyond $973 \mathrm{~K}$, the TL intensity is found to be decreased with the increase in heat treatment. The glow curves are analyzed by the glow curve deconvolution technique and trap parameters are estimated and discussed in this paper.
\end{abstract}

(C) 2008 Elsevier Ltd. All rights reserved.

Keywords: Aluminum oxide; Swift heavy ions; Thermoluminescence; Trap parameters

\section{Introduction}

Thermoluminescence (TL) is a well-established and very sensitive technique for estimating the concentration of luminescent centers in solids. The principle of TL process is discussed in detail elsewhere (Chen and McKeever, 1997; Furetta, 2003; Lakshminarasappa et al., 1992). TL yield gives information about the concentration of luminescent centers, whereas TL emission reveals the nature of traps (defect centers) created in solids. TL yield and the shape of TL glow curves vary with the amount and nature of ionizing radiation used for excitation of materials (Gieb et al., 1998; Lakshminarasappa et al., 1992). The effect of ion beam on the materials depends on the ion energy, fluence and ion species. When an energetic ion penetrates the material, it loses its kinetic energy in two ways: (i) nuclear stopping and (ii) electronic stopping. The nuclear stopping is dominant for low-energy ions and the energy lost in this process is called nuclear energy loss $\left(S_{\mathrm{n}}\right)$. The other mode of energy loss in matter is by exciting or ionizing the atom by

\footnotetext{
* Corresponding author. Tel.: +91 8022961486 , mobile: +9194495 26648; fax: +91 8023219295

E-mail address: bnlnarasappa@gmail.com (B.N. Lakshminarasappa).
}

inelastic collisions and the energy spent in this process is called electronic energy loss $\left(S_{\mathrm{e}}\right)$. Electronic stopping is dominant for ions with high energies, where the displacement of atoms due to elastic collisions is insignificant (Horowitz et al., 2001). Swift heavy ions (SHI) are very useful for modifying the properties of thin films, foils, surfaces of bulk solids and nanostructured materials. SHI passing through a material cause intense electronic excitations along the ion trajectory and result in defect production and phase transformation on a nanometer scale. Investigation on properties of such materials will throw light for controlled modification of some technological important materials for application in the field of biology, nuclear science and material science. However, the use of energetic ion beams bringing about modifications in some important materials has become a wide field of basic research as well as a powerful technique for several studies and applications (Abdel-Hamid et al., 1997; Salah and Sahare, 2006).

Aluminum oxide $\left(\alpha-\mathrm{Al}_{2} \mathrm{O}_{3}\right)$, one of the standard materials for the science of ceramics, is known as a radiation-resistant material, which can be used under various radiation environments. It possesses high melting point, high thermal conductivity and high electrical resistance even at elevated temperatures. Hence, $\alpha-\mathrm{Al}_{2} \mathrm{O}_{3}$ is considered as a technologically important material 
for diverse applications, such as solid-state lasers, UV windows and more recently, fusion energy devices. Furthermore, alumina is suggested as efficient and reproducible TL dosimeters for both ultraviolet light and ionizing radiation (Dalmasso et al., 2006; Summers, 1984). In the present investigation, TL behavior of $\mathrm{Al}_{2} \mathrm{O}_{3}$ with high energetic swift $\mathrm{Au}^{9+}$ ion irradiation has been studied and the results are presented.

\section{Material and methods}

Polycrystalline aluminum oxide used in the present investigations was synthesized by combustion technique based on the procedure described in detail elsewhere (Kingsley and Patil, 1988). In order to handle the phosphor easily, $100 \mathrm{mg}$ of polycrystalline $\mathrm{Al}_{2} \mathrm{O}_{3}$ powder was grained into a fine powder using an agate and mortar and mixed with a drop of polyvinyl alcohol in stainless steel die to make pellets. Pellets of $6 \mathrm{~mm}$ diameter and $1 \mathrm{~mm}$ thickness from the above mixture were obtained by pressing at about $70 \mathrm{MPa}$ using a home-made pelletizer at room temperature. One of these samples was used as pristine for comparison with those irradiated ones. A series of the pellets were annealed in a tubular furnace at different temperatures in the range of $773-1173 \mathrm{~K}$ for $3 \mathrm{~h}$ in oxygen atmosphere. As-prepared pellets and annealed samples were irradiated with $120 \mathrm{MeV}$ swift $\mathrm{Au}^{9+}$ ions for various fluences in the range of $1 \times 10^{11}-2 \times 10^{13}$ ions $^{-2}{ }^{-2}$ using 15UD Tandem Pelletron Accelerator in Materials Science beam line at the Inter University Accelerator Centre, New Delhi, India. The range and energy losses in aluminum oxide were calculated using the TRIM-code (SRIM-2003; www.srim.org). The range of $120 \mathrm{MeV}$ gold ions in aluminum oxide is estimated to be $9.18 \mu \mathrm{m}$ and $S_{\mathrm{e}}=2.483 \times$ $10^{04} \mathrm{eV} \mathrm{nm}^{-1}$ and $S_{\mathrm{n}}=3.608 \times 10^{02} \mathrm{eV} \mathrm{nm}^{-1}$.

TL measurements were made using Harshaw TL analyzer, model 3500, connected to a PC to record and process the experimental data. The TL signal was integrated from RT up to $673 \mathrm{~K}$ at a heating rate of $10 \mathrm{~K} \mathrm{~s}^{-1}$. The trap parameters were obtained using only the glow peak shape method (modified by Chen; Horowitz, 1984), i.e. deconvolution of glow curves is done first, using commercially available Origin 6.1 software. Then, the trap parameters are calculated manually using the equations suggested by Chen. When compared with other methods such as the initial rise method, $T_{\mathrm{m}}-T_{\text {stop }}$ method, etc. in the estimation of the trap parameters, glow curve shape method (modified by Chen; Horowitz, 1984) is known to be the best method and has less error.

\section{Results and discussion}

\subsection{Analysis of TL glow curves}

Fig. 1 shows the TL glow curves of combustion-synthesized aluminum oxide irradiated with $120 \mathrm{MeV} \mathrm{Au}{ }^{9+}$ ions for fluences in the range of $1 \times 10^{11}-2 \times 10^{13}$ ions $\mathrm{cm}^{-2}$. Two TL glows-a well-resolved one with peak at $\sim 623 \mathrm{~K}\left(\mathrm{Tg}_{2}\right)$ and an unresolved one at $\sim 513 \mathrm{~K}(\mathrm{Tg} 1)$ - are recorded at a heating rate $(\beta)$ of $10 \mathrm{~K} \mathrm{~s}^{-1}$. The primary defect centers generated in $\mathrm{Al}_{2} \mathrm{O}_{3}$ due to ionizing radiation are reported to be F-centers

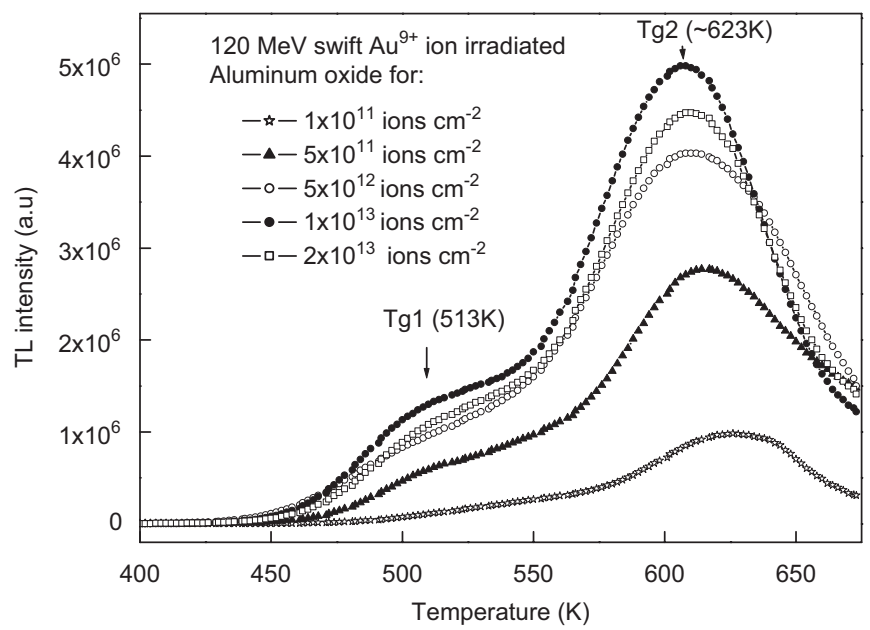

Fig. 1. Thermoluminescence glow curves of combustion synthesized $120 \mathrm{MeV}$ swift $\mathrm{Au}^{9+}$ ion irradiated aluminum oxide.

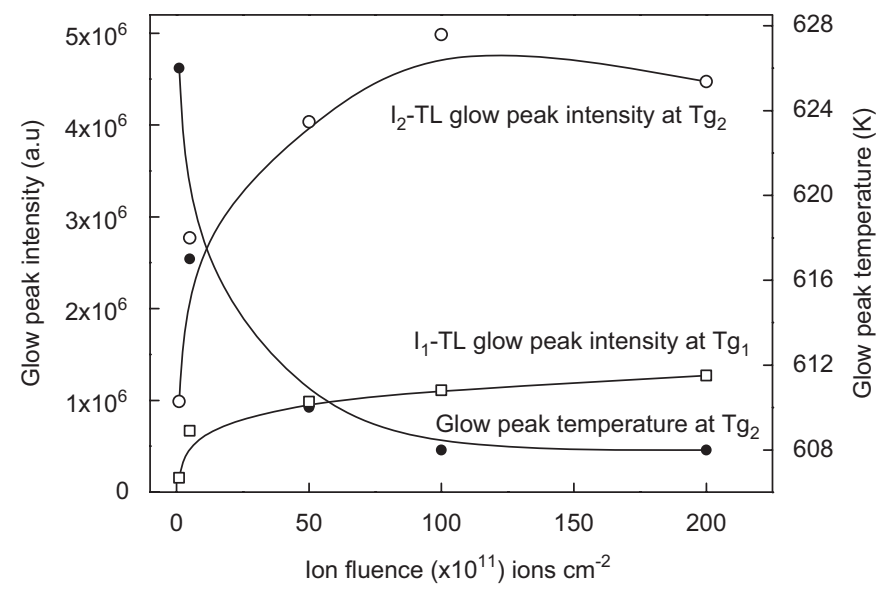

Fig. 2. Variation of TL glows peak $\left(I_{1}\right.$ and $\left.I_{2}\right)$ intensity and prominent glow peak temperature $\left(\mathrm{Tg}_{2}\right)$ with fluence.

and hole centers (McKeever et al., 1999). F-centers are found to be enhanced with increase in ionizing radiation and consequently the enhancement in TL intensity is also observed. The enhancement in TL intensity due to high concentration of luminescence centers (F-centers) caused by increasing the ionizing radiation is observed. The prominent TL glow peak observed at $\sim 623 \mathrm{~K}$ in the present study is attributed to Fcenters. During ion bombardment, in addition to displacements of atoms/ions, other consequences involving the coordination behavior of atoms may take place. These are known as thermal spikes resulting from sudden heating during $10^{-12}-10^{-10} \mathrm{~s}$ (Nagabhushana et al., 2003).

TL glow curve analysis leads to the estimation (see Fig. 4) of localized trap depth. The analysis also leads to the estimation of the frequency factor (s), which gives information about the electrons that are released from the trap due to thermal energy, that is the released electrons may get retrapped at the trapping center, which is known as second-order kinetics. On the other hand, the thermally released electrons may reach the conduction 
Table 1

Kinetic parameters of as-prepared aluminum oxide irradiated with swift $\mathrm{Au}^{9+}$ ions, obtained by using the glow curve shape method (modified by Chen)

\begin{tabular}{|c|c|c|c|c|c|c|c|c|c|}
\hline \multirow[t]{2}{*}{ Ion fluence (ions $\mathrm{cm}^{-2}$ ) } & \multirow[t]{2}{*}{ TL glow curve } & \multirow[t]{2}{*}{$T_{\mathrm{m}}(\mathrm{K})$} & \multirow[t]{2}{*}{$\mu_{\mathrm{g}}$} & \multirow[t]{2}{*}{$b$} & \multicolumn{4}{|c|}{ Activation energy (eV) } & \multirow[t]{2}{*}{$s\left(\mathrm{~s}^{-1}\right)$} \\
\hline & & & & & $E_{\tau}$ & $E_{\delta}$ & $E_{\omega}$ & $E_{\text {ave }}$ & \\
\hline \multirow[t]{2}{*}{$1 \times 10^{11}$} & $\mathrm{~A}$ & 557.07 & 0.504 & 2 & 0.936 & 0.993 & 0.969 & 0.966 & $1.882 \times 10^{08}$ \\
\hline & B & 627.72 & 0.504 & 2 & 1.069 & 1.613 & 1.618 & 1.613 & $3.593 \times 10^{07}$ \\
\hline \multirow[t]{2}{*}{$5 \times 10^{11}$} & A & 518.16 & 0.507 & 2 & 1.277 & 1.284 & 1.287 & 1.283 & $1.658 \times 10^{12}$ \\
\hline & $\mathrm{B}$ & 620.03 & 0.500 & 2 & 0.957 & 1.034 & 1.000 & 0.997 & $9.887 \times 10^{08}$ \\
\hline \multirow[t]{2}{*}{$5 \times 10^{12}$} & A & 509.08 & 0.515 & 2 & 0.975 & 1.102 & 1.098 & 1.058 & $1.133 \times 10^{10}$ \\
\hline & B & 612.77 & 0.502 & 2 & 0.963 & 1.034 & 1.115 & 1.037 & $1.027 \times 10^{09}$ \\
\hline \multirow[t]{2}{*}{$1 \times 10^{13}$} & $\mathrm{~A}$ & 513.64 & 0.503 & 2 & 1.101 & 1.141 & 1.113 & 1.118 & $4.481 \times 10^{10}$ \\
\hline & B & 606.94 & 0.504 & 2 & 1.175 & 1.218 & 1.205 & 1.119 & $6.348 \times 10^{09}$ \\
\hline \multirow[t]{2}{*}{$2 \times 10^{13}$} & A & 515.85 & 0.505 & 2 & 1.178 & 1.185 & 1.192 & 1.185 & $4.395 \times 10^{09}$ \\
\hline & B & 610.04 & 0.504 & 2 & 1.102 & 1.162 & 1.125 & 1.123 & $6.660 \times 10^{09}$ \\
\hline
\end{tabular}

band without getting retrapped and it is known as first-order kinetics. Further, it has been observed that the glow curves are more symmetric in nature on a wide temperature scale. This is one of the characteristic features of a second-order TL glow curve and this is due to the fact that in a second-order reaction significant concentrations of released electrons are getting retrapped before they recombine with hole centers. This might be the reason for the delay in the luminescence emission and hence spreading out of the emission over a wider temperature range (Chen and McKeever, 1997).

Fig. 2 shows the variation of TL glow peak intensities $\left(I_{1}\right.$ and $\left.I_{2}\right)$ and also prominent glow peak temperature $\left(\mathrm{Tg}_{2}\right)$ as a function of ion fluence in $\mathrm{Au}^{9+}$ ion irradiated aluminum oxide. It is found that the intensity at the TL glow peaks increases with the increase in ion fluence up to $\sim 1 \times 10^{13}$ ions cm $\mathrm{cm}^{-2}$ and thereafter it reaches saturation. This might be correlated to the saturation of defects created due to very high local dose around the ion path. Gieb et al. (1998) observed that, for a given ion energy, the luminescence efficiency was found to decrease with the decrease in projectile charge $(Z)$ due to the ionization, which is proportional to the square of the effective projectile charge $\left(Z_{\text {eff }}^{2}\right)$. Also, it is found that the glow peak temperature $\mathrm{Tg}_{2}$ shifts towards the lower temperature region with the increase in ion fluence. This may be attributed to the increase in concentrations of trapped electrons $\left(n_{0}\right)$ (F-centers) in lattice. According to Garlik and Gibson (Chen and McKeever, 1997) for a second-order TL glow peak, as $n_{0}$ increases, so too does $s$, (in good agreement with the calculated values of $s$ given in Table 1), which in turn causes the TL peak to shift towards the lower temperature side.

TL glow curves of combustion-synthesized and heat-treated aluminum oxide irradiated with $120 \mathrm{MeV}$ swift $\mathrm{Au}^{9+}$ ions for the fluence of $1 \times 10^{12}$ ions $\mathrm{cm}^{-2}$ are given in Fig. 3. Here, all the samples including pristine are irradiated at the same time and for the same fluence. The glow curves indicate a dominant peak at $\sim 615 \mathrm{~K}$ with a shoulder on all curves at $\sim 510 \mathrm{~K}$. It is found that the TL intensity increases with heat treatment when compared with that in as-prepared sample. However, the

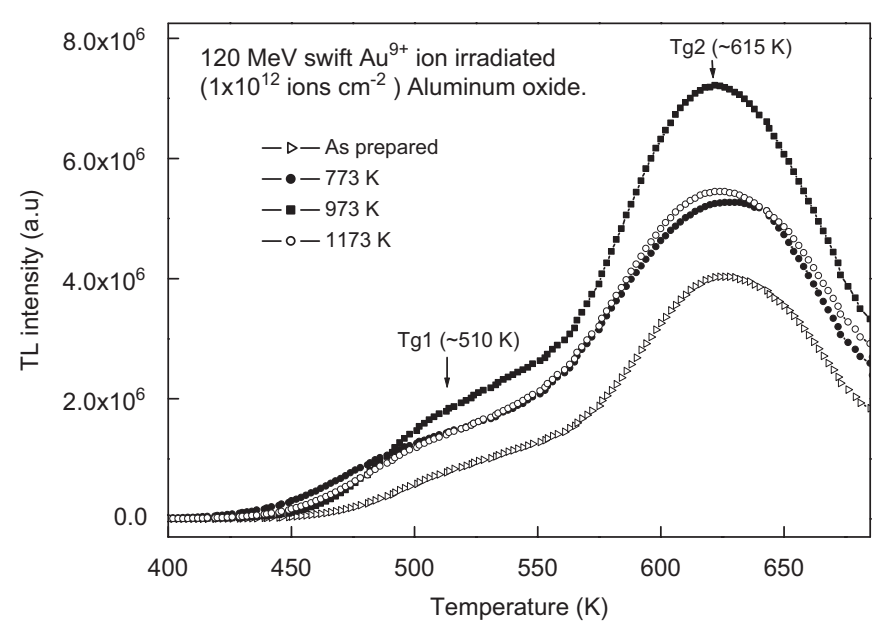

Fig. 3. Thermoluminescence glow curves of combustion-synthesized heat-treated $120 \mathrm{MeV}$ swift $\mathrm{Au}^{9+}$ ion irradiated $\left(1 \times 10^{12}\right.$ ions $\left.\mathrm{cm}^{-2}\right)$ aluminum oxide.

TL intensity is found to increase up to $973 \mathrm{~K}$. But, beyond $973 \mathrm{~K}$, the effect of heat treatment reduces the TL intensity as can be seen from the figure for the case of $1173 \mathrm{~K}$ heat treated one.

It may be noted that the TL glow peak temperature depends on various parameters such as nature of the sample (crystalline, semi-crystalline, non-crystalline, etc.), history of the sample (Pre-exposed to natural radiations, age of the sample, etc.), impurity content of the sample, heat treatment given to the sample prior to irradiation, nature of ionizing radiation, amount of irradiation (dose/fluence), temperature at which the sample was irradiated, temperature at which TL measurements are made, time gap between measurements, environment of the sample during experiments (humidity, atmospheric gas, etc.) type of detector, heating rate, etc. Hence, there exists the difference in TL intensity with the amount of irradiation dose and also with the degree of thermal treatment given to the samples prior to irradiation. 


\subsection{Calculation of kinetic parameters}

The evaluation of kinetic parameters known as trapping parameters, i.e. activation energy $(E)$ of the traps involved in TL emission, order of kinetics $(b)$ and frequency factor $(s)$ associated with the glow peaks of TL, is one of the important aspects of studies in condensed matter physics. Any complete description of the TL characteristics of TL material requires the knowledge of these parameters. Here, $E$ is a measure of the energy required to eject an electron from the defect center to the conduction band and $S$ is the rate of electron ejection. The order of kinetics $b$ is a measure of the probability that a free electron gets retrapped. This retrapping effect also depends on the availability of empty traps. The retrapping effect increases with the density of empty traps.

To obtain these parameters, the glow curves are deconvoluted using Origin 6.1 software. The trap parameters of deconvoluted curves are calculated using the glow curve shape method (modified by Chen). To determine the order of kinetics, the form factor (symmetry factor)

$\mu_{\mathrm{g}}\left\{\mu_{\mathrm{g}}=\frac{T_{2}-T_{\mathrm{m}}}{T_{2}-T_{1}}\right\}$,

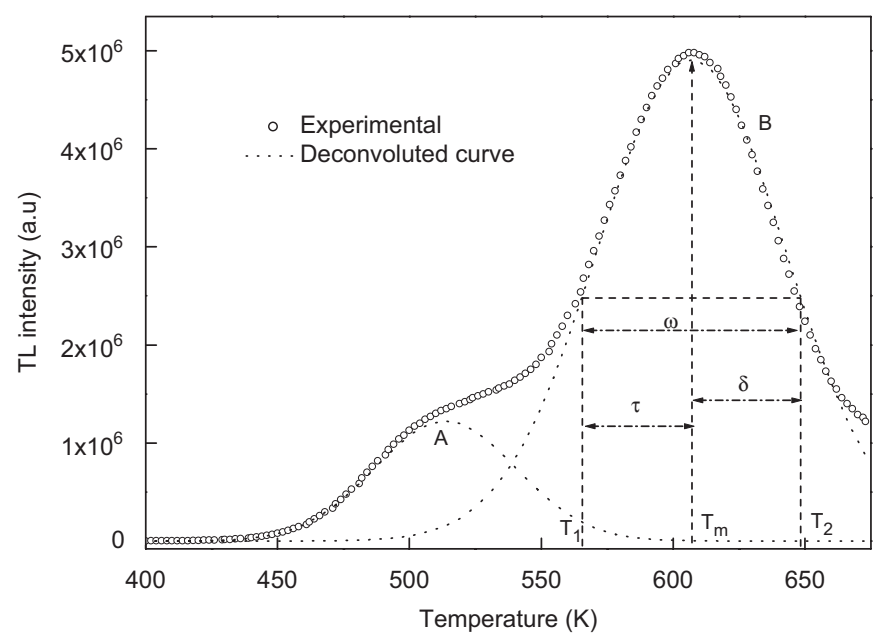

Fig. 4. Thermoluminescence deconvoluted glow curve of combustion-synthesized $120 \mathrm{MeV}$ swift $1 \times 10^{13} \mathrm{Au}^{9+}$ ion irradiated aluminum oxide. which involves $T_{1}$ and $T_{2}$ is calculated ( $T_{1}$ and $T_{2}$ are the temperatures corresponding to the half of the maximum intensities on either side of the glow peak maximum $-T_{\mathrm{m}}$ ). Theoretically, the form factor that ranges between 0.42 and 0.52 is close to 0.42 for first-order kinetics and 0.52 for second-order kinetics (Horowitz et al., 2001).

The equations used in the glow curve shape method (modified by Chen) (Horowitz, 1984) can be summarized as follows:

The activation energy $(E)$

$E_{\alpha}=c_{\alpha}\left(\frac{k T_{\mathrm{m}}^{2}}{\alpha}\right)-b_{\alpha}\left(2 k T_{\mathrm{m}}\right)$

where $\alpha=\tau, \delta$, $\omega$ with $\tau=T_{\mathrm{m}}-T_{1}, \delta=T_{2}-T_{\mathrm{m}}, \omega=T_{2}-T_{1}$

$c_{\tau}=1.51+3.0\left(\mu_{\mathrm{g}}-0.42\right), \quad b_{\tau}=1.58+4.2\left(\mu_{\mathrm{g}}-0.42\right)$

$c_{\delta}=0.976+7.3\left(\mu_{\mathrm{g}}-0.42\right), \quad b_{\delta}=0$

$c_{\omega}=2.52+10.2\left(\mu_{\mathrm{g}}-0.42\right), \quad b_{\omega}=1$.

The frequency factor $(s)$

$\frac{\beta E}{k T_{\mathrm{m}}^{2}}=s \exp \left(\frac{-E}{k T_{\mathrm{m}}}\right)\left[1+(b-1) \Delta_{\mathrm{m}}\right]$

where $\Delta_{\mathrm{m}}=2 k T_{\mathrm{m}} / E, b$ is the order of kinetics, $k$ is the Boltzmann constant $\left(8.6 \times 10^{-5} \mathrm{eV} \mathrm{K}^{-1}\right)$ and $\beta$ is the linear heating rate $\left(10 \mathrm{~K} \mathrm{~s}^{-1}\right)$.

The deconvoluted TL glow curves and the experimental ones after deconvolution for as-prepared $\mathrm{Al}_{2} \mathrm{O}_{3}$ irradiated with the fluence of $1 \times 10^{13}$ ions $\mathrm{cm}^{-2}$ are shown in Fig. 4. Also, the values of $\tau, \delta, \omega$ (in Kelvin) used for the estimation of kinetic parameters are indicated in the diagram. The experimental glow curve was deconvoluted into two glows having the glow peak one at $\sim 513 \mathrm{~K}$ and the other dominant one at $\sim 607 \mathrm{~K}$.

The kinetic parameters of as-prepared aluminum oxide irradiated with swift $\mathrm{Au}^{9+}$ ions for various fluences obtained by using the glow curve shape method (modified by Chen) are given in Table 1. Table 2 gives the kinetic parameters of annealed aluminum oxide irradiated with swift $\mathrm{Au}^{9+}$ ions for the fluence of $1 \times 10^{12}$ ions $\mathrm{cm}^{-2}$. From the data, it is obvious that a considerable amount of retrapping takes place in all

Table 2

Kinetic parameters of annealed aluminum oxide irradiated with swift $\mathrm{Au}^{9+}$ ions for the fluence of $1 \times 10^{12}$ ions $\mathrm{cm}^{-2}$, obtained by using the glow curve shape method (modified by Chen)

\begin{tabular}{|c|c|c|c|c|c|c|c|c|c|}
\hline Annealing temperature & TL glow curve & $T_{\mathrm{m}}(\mathrm{K})$ & $\mu_{\mathrm{g}}$ & $b$ & \multicolumn{4}{|c|}{ Activation energy (eV) } & $s\left(\mathrm{~s}^{-1}\right)$ \\
\hline As prepared & A & 521.89 & 0.532 & 2 & 1.138 & 1.169 & 1.181 & 1.163 & $8.262 \times 10^{10}$ \\
\hline \multirow[t]{2}{*}{$773 \mathrm{~K}$} & A & 507.32 & 0.514 & 2 & 0.765 & 0.822 & 0.797 & 0.795 & $2.651 \times 10^{07}$ \\
\hline & B & 625.95 & 0.502 & 2 & 0.858 & 0.955 & 0.915 & 0.909 & $5.198 \times 10^{06}$ \\
\hline \multirow[t]{2}{*}{$1173 \mathrm{~K}$} & A & 506.00 & 0.517 & 2 & 1.102 & 0.923 & 1.027 & 0.987 & $2.919 \times 10^{09}$ \\
\hline & $\mathrm{B}$ & 624.20 & 0.501 & 2 & 0.917 & 0.898 & 0.853 & 0.889 & $3.686 \times 10^{06}$ \\
\hline
\end{tabular}


second-order peaks. It is believed that there are some deep and shallow traps and for this reason there could be retrapping of the electrons at deep traps going to upper shallow traps by stimulation due to thermal energy. The competition among them might be giving various releasing and retrapping probabilities, which might have resulted in different frequency factors. The traps could be either electron traps or hole traps or of both kinds (Salah et al., 2004). However, it should be mentioned that this point needs more detailed investigations using other techniques such as thermostimulated conductivity, TL emission, electron spin resonance, photoluminescence, photoacoustic studies/optical absorption, etc., in order to understand the TL mechanism, trapping and recombination centers associated with thermostimulated light emission of aluminum oxide due to SHI.

\section{Conclusions}

The $120 \mathrm{MeV}$ swift $\mathrm{Au}^{9+}$ irradiated aluminum oxide exhibits a strong TL glow peak at $\sim 626 \mathrm{~K}$ with shoulder at $\sim 513 \mathrm{~K}$. Deconvolution revealed that both TL peaks show second-order kinetics. The TL intensity is found to increase with the increase in ion fluence up to $1 \times 10^{13}$ ions $\mathrm{cm}^{-2}$ and thereafter it saturates and then decreases. Also, it is found that the prominent glow peak temperature $\left(\mathrm{Tg}_{2}\right)$ shifts towards the lower temperature region with the increase in ion fluence. The TL glow peak intensity is found to be enhanced in the case of heat-treated samples when compared with as-prepared samples.

\section{Acknowledgments}

The authors express their sincere thanks to Dr. D.K. Avasthi and Dr. S.P. Lochab, Inter University Accelerator Centre, New Delhi, for many useful discussions, constant encouragement and help during the experiment. Also, K.R.N is grateful to the Inter University Accelerator Centre, New Delhi, for providing fellowship under the UFUP (No. 37314) scheme.

\section{References}

Abdel-Hamid, H.M., Hussein, M.A., Abou-Zeid, H.M., Morsy, S.M., 1997. Effect of ion bombardment on the Tl-response of both LiF (TLD-100) and a home-made LiF:Mg, Cu,P phosphors. Nucl. Instrum. Methods B 132, 119-122.

Chen, R., McKeever, S.W.S., 1997. Theory of Thermoluminescence and Related Phenomenon. World Scientific, Singapore. pp. 29-33.

Dalmasso, C., Iacconi, P., Beauvy, M., Lapraz, D., Balan, E., Calas, G., 2006. Radiation damage induced by krypton ions in sintered $\alpha-\mathrm{Al}_{2} \mathrm{O}_{3} \mathrm{C}$. Radiat. Prot. Dosim. 119, 222-225.

Furetta, C., 2003. Handbook of Thermoluminescence. World Scientific, Singapore.

Gieb, O.B., Kramer, M., Kraft, G., 1998. Efficiency of thermoluminescent detectors to heavy charged particles. Nucl. Instrum. Methods. B 142, 592-598.

Horowitz, Y.S., 1984. Thermoluminescence and Thermoluminescent Dosimetry, vol. 1. Chemical Rubber Co., Boca Raton.

Horowitz, Y.S., Avila, O., Rodriguez-Villafuerte, M., 2001. Theory of heavy charged particle response (efficiency and supralinearity) in TL materials. Nucl. Instrum. Methods B 184, 85-112.

Kingsley, J.J., Patil, K.C., 1988. A novel combustion process for the synthesis of fine particle $\alpha$-alumina and related oxide materials. Mater. Lett. 6, 427-432.

Lakshminarasappa, B.N., Devaraj, N., Murti, Y.V.G.S., 1992. Thermoluminescence of $\mathrm{X}$-irradiated $\mathrm{NaBr}$ crystals. In: Proccedings of National Seminar on Thermoluminescence and its Applications. Tata McGraw-Hill, India, 250pp.

McKeever, S.W.S., Akselrod, M.S., Colyott, L.E., Larsen, N.A., Polf, J.C., Whitley, V., 1999. Characterization of $\mathrm{Al}_{2} \mathrm{O}_{3}$ for use in thermally and optically stimulated luminescence dosimetry. Radiat. Protect. Dosim. 84, 163-168.

Nagabhushana, H., Lakshminarasappa, B.N., Fouran Singh, Avasthi, D.K., 2003. Thermoluminescence studies of $\mathrm{Si}^{8+}$ ion irradiated Kyanite. Radiat. Meas. 36, 653-655.

Salah, N., Sahare, P.D., 2006. The influence of high-energy 7Li ions on the TL response and glow curve structure of $\mathrm{CaSO}_{4}$ : Dy. J. Phys. D: Appl. Phys. 39, 2684-2691.

Salah, N., Sahare, P.D., Nawaz, S., Lochab, S.P., 2004. Luminescence characteristics of $\mathrm{K}_{2} \mathrm{Ca}_{2}\left(\mathrm{SO}_{4}\right)_{3}$ : Eu, Tb micro- and nanocrystalline phosphor. Radiat. Eff. Defects Solid 159, 321-334.

Summers, G.P., 1984. Thermoluminescence in single crystal $\alpha-\mathrm{Al}_{2} \mathrm{O}_{3}$. Radiat. Prot. Dosim. 8, 69-80. 